\title{
Supermassive Black Holes as Giant Bose-Einstein Condensates
}

\author{
Theo M. Nieuwenhuizen \\ Institute for Theoretical Physics, University of Amsterdam, Valckenierstraat 65, 1018 XE Amsterdam, The Netherlands
}

PACS 04.70.Bw - Classical black holes

PACS $04.20 . \mathrm{Cv}$ - Fundamental problems and general formalism

PACS 04.20.Jb - Exact solutions

\begin{abstract}
The Schwarzschild metric has a divergent energy density at the horizon, which motivates a new approach to black holes. If matter is spread uniformly throughout the interior of a supermassive black hole, with mass $M \sim M_{\star}=2.3410^{8} M_{\odot}$, it may arise from a Bose-Einstein condensate of densely packed H-atoms. Within the Relativistic Theory of Gravitation with a positive cosmological constant, a bosonic quantum field is coupled to the curvature scalar. In the Bose-Einstein condensed groundstate an exact, selfconsistent solution for the metric is presented. It is regular with a specific shape at the origin. The redshift at the horizon is finite but large, $z \sim 10^{14} M_{\star} / M$. The binding energy remains as an additional parameter to characterize the $\mathrm{BH}$; alternatively, the mass observed at infinity can be any fraction of the rest mass of its constituents.
\end{abstract}

On the basis of the Schwarzschild, Kerr and KerrNewman metrics, it generally believed that black holes (BHs) are singular objects with all matter localized in the center or, if rotating, on an infinitely thin ring. Recent approaches challenge this unintuitive assumption and consider matter just spread throughout the interior. [1-3] Here we shall follow this line of research. To start, let us just look at some orders of magnitude. For solar mass neu' tron stars it is known that the density is about the nuclear ' density. Solar mass black holes are about 10 times smaller, thus 1000 times more compact. Clearly, this begs for a Quantum Chromodynamics description in curved space.

We shall focus on the other extreme, superheavy BHs. They occur in the center of each galaxy and weigh about $M_{\mathrm{BH}}=0.0012 M_{\text {bulge. }}$ [4] Let us assume that they consist of hydrogen atoms and that mass and particle number are related as $M \equiv \nu N m_{\mathrm{H}}$ with some $\nu \leq 1$. If we neglect rotation, the radius is $R=G M / c^{2}$ (see below). We may compare the $\mathrm{BH}$ density $3 N / 4 \pi R^{3}$ with the one of densely packed, non-overlapping $\mathrm{H}$-atoms, that is, with the Bohr density $n_{\mathrm{B}} \equiv 3 / 4 \pi a_{0}^{3}$, with $a_{0}=0.529 \AA$ 献 Bohr radius. This yields a mass $M_{\star}=c^{3}\left(a_{0}^{3} / G^{3} m_{\mathrm{H}}\right)^{1 / 2}$ (we take $\nu=1$ here), which lies in the range of observed supermassive black holes, $M_{\star}=4.6610^{38} \mathrm{~kg}=2.3410^{8} M_{\odot}$.

Next comes the question how matter can withstand the enormous pressure normally associated with such high densities. It was proposed originally by Sacharov that the vacuum equation of state $p=-\rho$ could describe matter at superhigh densities. [5] Laughlin and coworkers assume that matter near the horizon could be in its Bose-Einstein condensed (BEC) phase, modeled by the vacuum equation of state. [1] Dymnikova considers BHs obeying it in the interior, which, however, have one or two horizons. [2] Mazur and Mottola take the BEC idea over to the interior, and investigate a "gravo-star", of which the interior obeys the vacuum equation of state, and which is surrounded by a thin shell of normal matter having the stiff equation of state $p=+\rho$. This solution is regular everywhere. [3]

We shall demonstrate that a supermassive BH can exist as a self-gravitating hydrogen cloud, in a Bose-Einstein condensed phase. We study the problem in a series of improved starting points: assume a stiff equation of state, self-consistently solve a quantum field coupled to the curvature scalar, first for a uniform groundstate wavefunction and next for a space-dependent one. Hereto we have to employ the Relativistic Theory of Gravitation (RTG), which reproduces all weak gravitational effects in the solar system $[6,7]$ as well as the $\Lambda$ CDM cosmology [8].

We consider a static metric with spherical symmetry,

$$
\mathrm{d} s^{2}=U(r) c^{2} \mathrm{~d} t^{2}-V(r) \mathrm{d} r^{2}-W^{2}(r)\left(\mathrm{d} \theta^{2}+\sin ^{2} \theta \mathrm{d} \phi^{2}\right)
$$

The gravitational energy density arises from the LandauLifshitz pseudo-tensor, [9] generalized to become a tensor in Minkowski space. $[8,10]$ For (1) it takes the form

$$
t^{00}=\frac{c^{4} W^{2}}{8 \pi G r^{6}}\left(-\frac{r^{2} V^{\prime} W W^{\prime}}{V}+r^{3} V^{\prime}-5 r^{2} W^{\prime 2}\right.
$$




$$
\left.+\frac{2 r^{3} V W^{\prime}}{W}+8 r W W^{\prime}-2 r^{2} V-3 W^{2}\right) .
$$

Let us start with the General Theory of Relativity (GTR). The Schwarzschild metric reads in the harmonic gauge

$$
U_{S}=\frac{1}{V_{S}}=1-\frac{2 M}{W_{S}}=\frac{r-M}{r+M}, \quad W_{S}=r+M .
$$

(We put $G=c=\hbar=1$.) It is singular at the horizon $r_{h}=M$ and involves the gravitational energy density

$$
t^{00}=\frac{1}{4 \pi r^{2}} \frac{\mathrm{d}}{\mathrm{d} r} \frac{M(r+M)^{3}(2 r+M)}{2 r^{3}(r-M)} .
$$

Its quadratic divergence at $r_{h}$ presents a hitherto overlooked peculiarity, that induces a negative infinite contribution to the total energy. For this reason, we shall switch to RTG with matter not located at the singularity $r=0$, but just spread out within the horizon.

Quantum field theory of Bose-Einstein condensed black holes. $\quad$ Let our $\mathrm{H}$-atoms be described by a bosonic field

$$
\hat{\psi}(\mathbf{r}, t)=\sum_{i} \hat{a}_{i} \psi_{i}(\mathbf{r}) e^{-i E_{i} t},
$$

where $i=\{n, \ell, m\},\left[\hat{a}_{i}, \hat{a}_{j}^{\dagger}\right]=\delta_{i j}$ and eigenfunctions factor as $\psi_{i}(\mathbf{r})=\phi_{n}(r) Y_{\ell m}(\theta, \phi)$. The rotating wave approximation then leads to the Lagrangian [11]

$$
L_{\mathrm{mat}}=g^{\mu \nu} \partial_{\mu} \hat{\psi}^{\dagger} \partial_{\nu} \hat{\psi}-\left(m^{2}+\xi R\right) \hat{\psi}^{\dagger} \hat{\psi}-\frac{\lambda}{4} \hat{\psi}^{\dagger 2} \hat{\psi}^{2} .
$$

For a field in curved space the renormalization group generates a coupling to the Ricci curvature scalar $R$. [12] Its strength $\xi$ is for now a phenomenological parameter. The dimensionless coupling $\lambda=8 m^{2} \mathrm{cg} / \hbar^{3}$ with $g=4 \pi \hbar^{2} a_{s} / m$ models the two particle interaction by the scattering length. For hydrogen in flat space one has [13]

$a_{s}=0.32 a_{0} \quad$ singlet state, $\quad a_{s}=1.34 a_{0}$ triplet.

We shall continue with the singlet value $\lambda=0.8110^{7}$.

With $\Psi_{0}=\left(2 E_{0} N_{0}\right)^{1 / 2} \psi_{0} e^{-i E_{0} t}$ for $N_{0}$ groundstate atoms, the relativistic Gross-Pitaevskii equation reads

$$
\left(\frac{1}{\sqrt{-g}} \partial_{\mu} \sqrt{-g} g^{\mu \nu} \partial_{\nu}+m^{2}+\xi R+\frac{\lambda\left|\Psi_{0}^{2}\right|}{4 E_{0}}\right) \Psi_{0}=0 .
$$

A homogeneous ground state, $\Psi_{0}(r, t)=\Psi_{0} e^{-i E_{0} t}$ occurs when

$$
-\frac{E_{0}^{2}}{U}+m^{2}+\xi R+\frac{\lambda}{4 E_{0}}\left|\Psi_{0}\right|^{2}=0 .
$$

We focus on RTG, which describes gravitation as a field in Minkowski space $[6,7]$ and posesses the same gravitational energy momentum tensor and thus also the gravitational energy density Eq. (2). [8] It extends the Hilbert-Einstein action with the cosmological term and a bimetric coupling between the Minkowski $(\gamma)$ and Riemann $(g)$ metrics,

$$
L=-\frac{R}{16 \pi}-\rho_{\Lambda}+\frac{1}{2} \rho_{\mathrm{bi}} \gamma_{\mu \nu} g^{\mu \nu}+L_{\mathrm{mat}} .
$$

(For $\rho_{\mathrm{bi}}=0$ it is just a field theory for GTR.) One has

$$
\begin{aligned}
& \rho_{\mathrm{tot}}=\rho+\rho_{\Lambda}+\frac{\rho_{\mathrm{bi}}}{2 U}-\frac{\rho_{\mathrm{bi}}}{2 V}-\frac{\rho_{\mathrm{bi}} r^{2}}{W^{2}}, \\
& p_{i}^{\text {tot }}=p_{i}-\rho_{\Lambda}+\frac{\rho_{\mathrm{bi}}}{2 U}-\frac{\rho_{\mathrm{bi}}}{2 V}+\frac{\rho_{\mathrm{bi}} r^{2}}{W^{2}} .
\end{aligned}
$$

with $i=r, \theta, \phi$. The value $\rho_{\mathrm{bi}}=\rho_{\Lambda}$ is imposed to have a Minkowski metric in the absence of matter. One may fix them to the observed positive cosmological constant [8]. However, historically the opposite choice $\rho_{\Lambda}<0$ was considered and the cosmological data were described by an additional inflaton field. [7] So the sign of $\rho_{\mathrm{bi}}$ is not known yet; We show that solving a realistic black hole settles this issue. The new point of RTG is that $g_{00}=U$ can be very small. Despite the smallness of $\rho_{\mathrm{bi}}$, the $\rho_{\mathrm{bi}} / U$ terms become relevant near the horizon $[6,8]$, bringing

$$
R=-8 \pi T_{\mathrm{tot}}=8 \pi\left(-\rho+p_{r}+p_{\theta}+p_{\phi}+\frac{\rho_{\mathrm{bi}}}{U}\right) .
$$

To start, let us consider the stiff equation of state

$$
\rho=\frac{1}{2} \rho_{c}\left(\frac{U_{c}}{U}+1\right), \quad p_{i}=p \equiv \frac{1}{2} \rho_{c}\left(\frac{U_{c}}{U}-1\right),
$$

where $\rho_{c}=3 / 32 \pi M^{2}$. For $U_{c}=0$ it is the vacuum equation of state $p=-\rho=$ const. One gets

$$
R=8 \pi \frac{\rho_{c} U_{c}+\rho_{\mathrm{bi}}}{U}-16 \pi \rho_{c} .
$$

Eq. (7) has a solution due to the $\xi R$-term. The constant and $1 / U$ terms term imply,

$$
\xi=\xi_{0}\left(1+\frac{\lambda\left|\Psi_{0}\right|^{2}}{4 E_{0} m^{2}}\right), \quad E_{0}^{2}=8 \pi \xi\left(\rho_{c} U_{c}+\rho_{\mathrm{bi}}\right),
$$

respectively. The dimensionless parameter

$$
\xi_{0}=\frac{2}{3} m^{2} M^{2}=1.8010^{54}\left(\frac{M}{M_{\star}}\right)^{2},
$$

appears to be large, but since $R \sim 1 / M^{2}$ the combination $\xi R$ is just of order $m^{2}$, making its effect of order unity. The metric can be solved as below; we omit details.

Self-consistent field theory. Rather than imposing an equation of state, the material energy-momentum tensor $T_{m}^{\mu \nu}$ should be derived from first principles, i.e. from the quantum field theory for the $\mathrm{H}$-atoms. Its energy density reads, if we exclude the effect of the $\xi R$-term,

$$
\begin{aligned}
\rho_{m} & =\frac{\left\langle\partial_{t} \psi^{\dagger} \partial_{t} \psi\right\rangle}{U}+\frac{\left\langle\partial_{r} \psi^{\dagger} \partial_{r} \psi\right\rangle}{V}+\frac{\left\langle\partial_{\theta} \psi^{\dagger} \partial_{\theta} \psi\right\rangle}{W^{2}} \\
& +\frac{\left\langle\partial_{\phi} \psi^{\dagger} \partial_{\phi} \psi\right\rangle}{W^{2} \sin ^{2} \theta}+m^{2}\left\langle\psi^{\dagger} \psi\right\rangle+\frac{\lambda}{4}\left\langle\psi^{\dagger 2} \psi^{2}\right\rangle .
\end{aligned}
$$


The pressures $\left(p_{r}^{m}, p_{\theta}^{m}, p_{\phi}^{m}\right)$ have this shape with signature $(++----),(+-+---)$, and $(+--+--)$, respectively. Spherical symmetry will imply that $p_{\theta}^{m}=p_{\phi}^{m} \equiv p_{\perp}^{m}$. For a uniform groundstate $p_{m}$ is isotropic,

$$
\left(\rho_{m}, p_{m}\right)=\frac{1}{2}\left(\frac{E_{0}}{U} \pm \frac{m^{2}}{E_{0}}\right)\left|\Psi_{0}^{2}\right| \pm \frac{\lambda\left|\Psi_{0}^{4}\right|}{16 E_{0}^{2}} .
$$

They consist of a vacuum part $p=-\rho=$ const. and a stiff part $p=+\rho \sim 1 / U$, the types studied in [3] and above. In the non-relativistic $\left(E_{0}=m\right)$ and flat space $(U=1)$ limit, they reduce for $\lambda=0$ to $\rho_{m}=m c^{2}\left|\Psi_{0}^{2}\right|$ and $p_{m}=0$.

Because of the $\xi R$-term in (5), the Einstein equations embody a direct backreaction of matter on curvature, $G^{\mu \nu}=8 \pi\left(T_{m}^{\mu \nu}+T_{\Lambda}^{\mu \nu}+T_{\mathrm{bi}}^{\mu \nu}\right)-16 \pi \xi\left\langle\psi^{\dagger} \psi\right\rangle G^{\mu \nu}$. To connect to the standard notation, $G^{\mu \nu}=8 \pi T_{\text {tot }}^{\mu \nu}$, we define $T^{\mu \nu}$ by

$$
T_{\mathrm{tot}}^{\mu \nu}=\frac{T_{m}^{\mu \nu}+T_{\Lambda}^{\mu \nu}+T_{\mathrm{bi}}^{\mu \nu}}{1+B} \equiv T^{\mu \nu}+T_{\Lambda}^{\mu \nu}+T_{\mathrm{bi}}^{\mu \nu},
$$

with direct backreaction strength of matter on the metric

$$
B=16 \pi \xi\left\langle\psi^{\dagger} \psi\right\rangle=\frac{8 \pi \xi\left|\Psi_{0}^{2}\right|}{E_{0}} .
$$

For $\lambda=0$ the curvature scalar follows from (16) as

$$
R=\frac{8 \pi}{1+B}\left(\frac{E_{0}\left|\Psi_{0}^{2}\right|+\rho_{\mathrm{bi}}}{U}-\frac{2 m^{2}}{E_{0}}\left|\Psi_{0}^{2}\right|\right) .
$$

Solving Eq. (7), we find two relations and a consequence,

$$
B=1, \quad E_{0}^{2}=8 \pi \xi \rho_{\mathrm{bi}}, \quad\left|\Psi_{0}^{2}\right|=\frac{E_{0}}{8 \pi \xi}=\frac{\rho_{\mathrm{bi}}}{E_{0}} .
$$

(The GTR situation, reached by taking $\rho_{\mathrm{bi}} \rightarrow 0$ first, would not allow a meaningful solution.) The first identity expresses a $100 \%$ direct backreaction of matter on the metric. This motivates to introduce the parameters [8]

$$
\mu=\sqrt{16 \pi \rho_{\mathrm{bi}}}=\sqrt{2 \Lambda}, \quad \bar{\mu}=\mu M=7.9010^{-15} \frac{M}{M_{\star}} .
$$

Instead of searching a finite $U$, as for boson stars, [14] we assume a very small $U$ with $U(0)=0$, coded by $v$,

$$
U=\frac{1}{2} \mu^{2} v^{2} W^{2}
$$

In terms of the mass function $\mathcal{M}(r)$, defined by

$$
V=\frac{W^{\prime 2}}{1-2 \mathcal{M} / W},
$$

the 00 and 11 Einstein equations take the form

$$
\mathcal{M}^{\prime}=4 \pi W^{\prime} W^{2} \rho_{\text {tot }}, \quad \frac{W-2 \mathcal{M}}{2 U W^{2}} \frac{U^{\prime}}{W^{\prime}}-\frac{\mathcal{M}}{W^{3}}=4 \pi p_{\text {tot }}^{r} .
$$

The Ansatz (22) solves them and yields, due to (20),

$$
v=1, \quad \mathcal{M}=\frac{W}{4}+\frac{W^{3}}{16 M^{2}} \frac{2 m^{2} M^{2}}{3 \xi} .
$$

For the Schwarzschild black hole the horizon occurs when $\mathcal{M}=M$ for $W=2 M$. Concerning the outside metric, we will be close to that situation. This implies again that a mass $M$ corresponds to $\xi=2 m^{2} M^{2} / 3$.

Let us introduce the 'Riemann' variables $x$ and $y$ by

$$
x=\frac{W}{2 M}, \quad y=\sqrt{1-x^{2}},
$$

so that $U=2 \bar{\mu}^{2} x^{2}$. The $\rho_{\mathrm{bi}}$ terms in (8) violate general coordinate invariance and impose the harmonic gauge,

$$
\frac{U^{\prime}}{U}-\frac{V^{\prime}}{V}+4 \frac{W^{\prime}}{W}=\frac{4 r V}{W^{2}} .
$$

With (22), (23) and $\mathcal{M}=\frac{1}{2} M\left(x+x^{3}\right)$ from (25), it brings

$$
\frac{2 x^{\prime}}{x}-\frac{2 x^{\prime \prime}}{x^{\prime}}-\frac{2 x x^{\prime}}{1-x^{2}}+\frac{4 x^{\prime}}{x}=\frac{8 r x^{\prime 2}}{x^{2}\left(1-x^{2}\right)} .
$$

Going to the inverse function $r(x)$ makes it linear,

$$
x^{2}\left(1-x^{2}\right) r^{\prime \prime}+x\left(3-4 x^{2}\right) r^{\prime}=4 r .
$$

The solution is then remarkably simple,

$$
r=r_{1}\left(1+\frac{y}{\sqrt{5}}\right) x^{\sqrt{5}-1}(1+y)^{-\sqrt{5}}
$$

(The second independent solution with $\sqrt{5} \rightarrow-\sqrt{5}$ is singular.) This determines the metric function $V$,

$$
V=\frac{2 W^{\prime 2}}{y^{2}}=\frac{5 M^{2}}{2 r_{1}^{2}} x^{4-2 \sqrt{5}}(1+y)^{2 \sqrt{5}} .
$$

Putting these results together, it now follows that

$$
\rho=\frac{3}{64 \pi M^{2}}, \quad p=-\frac{3}{64 \pi M^{2}},
$$

as the $1 / U$ terms cancel due to the relation $E_{0}^{2}=8 \pi \xi \rho_{\mathrm{bi}}$. So, after all, we reproduce the vacuum equation of state.

To normalize $\left|\Psi_{0}\right|$, we need the $3 d$ volume element in the future time direction, $\mathrm{d} \Sigma^{\mu}=\mathrm{d} r \mathrm{~d} \theta \mathrm{d} \phi n^{\mu} \sqrt{-g_{3}} \equiv \delta_{0}^{\mu} \mathrm{d} \mathcal{V}$, set by the timelike unit vector $n^{\mu}=\delta_{0}^{\mu} / \sqrt{U}$ and $g_{3}=$ $-V W^{4} \sin ^{2} \theta$. This results in $\mathrm{d} \mathcal{V}=\mathrm{d} r \mathrm{~d} \Omega \sqrt{V / U} W^{2}$.

The general inner product [12] $\left(\psi_{1}, \psi_{2}\right)=-i \int \mathrm{d} \Sigma^{\mu} \times$ $\left(\psi_{1} \partial_{\mu} \psi_{2}^{*}-\psi_{2}^{*} \partial_{\mu} \psi_{1}\right)$ defines the orthonormality

$$
\left(\psi_{i}, \psi_{j}\right)=\left(E_{i}+E_{j}\right) \int \mathrm{d} \mathcal{V} \psi_{i} \psi_{j}^{*} \equiv \delta_{i j} .
$$

With $\mathrm{d} \mathcal{V}=\mathrm{d} y \mathrm{~d} \Omega 8 M^{3} /(\bar{\mu} v)$ it yields

$$
\left|\Psi_{0}^{2}\right|=2 E_{0} N_{0}\left|\psi_{0}^{2}\right|=\frac{N_{0} v \bar{\mu}}{32 \pi M^{3}},
$$

having proper groundstate occupation, $\int \mathrm{d} \mathcal{V}\left|\Psi_{0}\right|^{2}=N_{0}$. 
In Eq. (13) the correction term is of order

$$
\bar{\lambda} \equiv \frac{\lambda}{32 \pi m^{2} \xi}=\frac{3 \lambda}{64 \pi m^{4} M^{2}} \approx 7.5810^{-12} \frac{M_{\star}^{2}}{M^{2}} .
$$

These corrections seem relevant for BH's with masses $M \sim 2.7510^{-6} M_{\star} \sim 645 M_{\odot}$. Much less below $M_{\star}$ the hydrogen atoms will get ionized, calling for fermionic fields for protons and electrons, which by a BCS pairing can again undergo a BEC transition. This BCS-BEC scenario is beyond the aim of the present paper.

The exterior. At the horizon $r_{h} \approx r_{1}, y_{h} \ll 1$ one has

$$
U=2 \bar{\mu}^{2}, \quad V=\frac{5}{2}, \quad W=2 M, \quad W^{\prime}=\frac{1}{2} \sqrt{5} y_{h} .
$$

We have to connect this to the vacuum solution outside the BH. Well away from matter, the harmonic constraint brings the Schwarzschild shape (3), where $M \equiv \mathcal{M}\left(r_{h}\right)$ is the mass, essentially as observed at infinity. The values (35) are far from Schwarzschild's, even when $r$ is near $M$ (e.g., $\left.W_{S}^{\prime}=1\right)$. The problem nevertheless appears to be consistent. Near $r_{h}$ we need the deformation of the Schwarzschild metric which regularizes its singularity due to the bimetric coupling. [6,7] An elegant scaling form for small $\bar{\mu}$ was presented by us, [8],

$$
\begin{aligned}
r & =M \frac{1+\eta\left(e^{\xi}+\xi+\log \eta+2\right)}{1-\eta\left(e^{\xi}+\xi+\log \eta+2\right)}, \quad U=\eta e^{\xi}, \\
V & =\frac{e^{\xi}}{\eta\left(1+e^{\xi}\right)^{2}}, \quad W=\frac{2 M}{1-\eta e^{\xi}-\bar{\mu}^{2}\left(\xi+w_{0}\right)} .
\end{aligned}
$$

Here $\xi$ is the running variable and $\eta$ a small scale. For $\eta e^{\xi}=\mathcal{O}(1)$ it coincides with the Schwarzschild solution. Matching with the interior appears to be possible,

$$
e^{\xi_{h}}=\sqrt{5} \bar{\mu}, \quad \eta=\frac{2}{\sqrt{5}} \bar{\mu}, \quad W^{\prime}=e^{\xi_{h}}+\frac{\bar{\mu}^{2}}{\eta}=\frac{3}{2} \sqrt{5} \bar{\mu},
$$

implying $y_{h}=3 \bar{\mu}$. Taken together, the three regimes, interior, horizon and exterior, provide an exact solution of the problem. At the origin it exhibits the singularities

$$
U=\bar{U}_{1} r^{\gamma_{\mu}}, \quad V=\frac{1}{2} \gamma_{\mu}^{2} \bar{W}_{1}^{2} r^{\gamma_{\mu}-2}, \quad W=\bar{W}_{1} r^{\frac{1}{2} \gamma_{\mu}},
$$

where $\gamma_{\mu}=\frac{1}{2}(\sqrt{5}+1)$ is the golden mean. But if we take $W$ as the coordinate, we have in the interior the shape

$$
\mathrm{d} s^{2}=\frac{1}{2} \mu^{2} W^{2} \mathrm{~d} t^{2}-\frac{2 \mathrm{~d} W^{2}}{1-W^{2} / 4 M^{2}}-W^{2} \mathrm{~d} \Omega^{2},
$$

which is regular at its origin, with the term $2 \mathrm{~d} W^{2}$ coding the above singularities.

We may rewrite the exterior solution by eliminating $\xi$,

$$
\begin{aligned}
r & =M \frac{1+U+(2 \bar{\mu} / \sqrt{5})(\log U+2)}{1-U-(2 \bar{\mu} / \sqrt{5})(\log U+2)}, \\
V & =\frac{U}{(U+2 \bar{\mu} / \sqrt{5})^{2}}, \\
W & =\frac{2 M}{1-U+2 \bar{\mu}^{2}-\bar{\mu}^{2} \log \left(U / 2 \bar{\mu}^{2}\right)} .
\end{aligned}
$$

This describes the free space region $r \geq M$, where $2 \bar{\mu}^{2} \leq$ $U \leq 1+\mathcal{O}(\bar{\mu})$. At the cosmic scale $r \sim 1 / \mu$ Newton's law picks up the Yukawa-type factor $\cos \mu r$, due to the tachyonic nature of gravitation in RTG with $\rho_{\mathrm{bi}}>0$. [8]

The interior shape can also be expressed with $U$ as running variable, where it lies in the range $\left(0,2 \bar{\mu}^{2}\right)$. Due to Eq. (29) it also holds that

$$
W=2 M x=\frac{1}{\mu} \sqrt{2 U}, \quad y=\sqrt{1-\frac{U}{2 \bar{\mu}^{2}}} .
$$

The locus and the metric function $V$ are given by (29), with $r_{1} \approx M$, and (30), respectively.

Contributions to the energy. With the weight $\mathrm{d} \mathcal{V}$ given below (32), the standard expression for the energy, $\int \mathrm{d} \mathcal{V} \rho$ scales as $1 / \bar{\mu}$ and even diverges logarithmically at $r=0$. However, in RTG the energy is determined by Eq. (2). At the origin it diverges as $r^{\sqrt{5}-5}$, which is integrable. The gravitational energy inside the $\mathrm{BH}$ reads

$$
U_{\text {grav }, \text { int }}=4 \pi \int_{0}^{M} \mathrm{~d} r r^{2} t^{00}=-842.898 M .
$$

The total energy density reads $\Theta^{00}=t^{00}+V W^{4} \rho_{\text {tot }} / r^{4}$. We can calculate the material and the bimetric energy,

$$
\begin{aligned}
U_{\text {mat }} & =4 \pi \int_{0}^{M} r^{2} \mathrm{~d} r \frac{V W^{4}}{r^{4}} \frac{3}{64 \pi M^{2}}=169.431 M, \\
U_{\mathrm{bi}} & =4 \pi \int_{0}^{M} r^{2} \mathrm{~d} r \frac{V W^{4}}{r^{4}} \frac{1}{64 \pi M^{2} x^{2}}=686.466 \mathrm{M} .
\end{aligned}
$$

Together they make up for

$$
U_{\text {interior }}=U_{\text {grav, int }}+U_{\text {mat }}+U_{\mathrm{bi}}=13 M .
$$

The energy density in the skin layer first has a large positive and then a large negative part, due to the term $r^{3} V^{\prime}$. The integrated effect is obtained easily since, in the formulation of the Einstein equations in Minkowski space, the total energy density is a total derivative, [8] The region $r>M$ thus yields $U_{\text {exterior }}=-12 M$. Together with the interior it confirms the total energy $U=M c^{2}$, expected from the decay of the metric, $g_{00}=1-2 G M / c^{2} r$.

Non-uniform groundstate. Till now we assumed that the groundstate wavefunction has a constant amplitude, and drops to zero at the horizon. Clearly, this cannot be exact. Taking $\Psi_{0} \rightarrow \Psi_{0}(r)$, we first take into account that in deriving the Einstein equations, partial integrations are to be performed. This brings derivatives of $B \sim\left|\Psi_{0}\right|^{2}$, and induces an extra term $T_{B}^{\mu \nu}$,

$$
(1+B) G_{\mu \nu}=8 \pi\left(T_{m}^{\mu \nu}+T_{\Lambda}^{\mu \nu}+T_{\mathrm{bi}}^{\mu \nu}+T_{B}^{\mu \nu}\right) .
$$

The elements of $\left(T_{B}\right)_{\nu}^{\mu} \equiv \operatorname{diag}\left(\rho_{B},-p_{r}^{B},-p_{\perp}^{B},-p_{\perp}^{B}\right)$ are

$$
\begin{aligned}
\rho_{B} & =\frac{1}{8 \pi}\left(\frac{B^{\prime \prime}}{V}+\frac{2 r B^{\prime}}{W^{2}}-\frac{B^{\prime} U^{\prime}}{2 U V}\right), \\
p_{r}^{B} & =\frac{-1}{8 \pi}\left(\frac{B^{\prime} U^{\prime}}{2 U V}+\frac{2 B^{\prime} W^{\prime}}{V W}\right), \\
p_{\perp}^{B} & =\frac{-1}{8 \pi}\left(\frac{B^{\prime \prime}}{V}+\frac{2 r B^{\prime}}{W^{2}}-\frac{B^{\prime} W^{\prime}}{V W}\right) .
\end{aligned}
$$


Eq. (43) now leads to a total energy momentum tensor

$T_{\mathrm{tot}}^{\mu \nu}=\frac{T_{m}^{\mu \nu}+T_{\Lambda}^{\mu \nu}+T_{\mathrm{bi}}^{\mu \nu}+T_{B}^{\mu \nu}}{1+B} \equiv T^{\mu \nu}+T_{\Lambda}^{\mu \nu}+T_{\mathrm{bi}}^{\mu \nu}$.

$T_{\nu}^{\mu} \equiv \operatorname{diag}\left(\rho,-p_{r},-p_{\perp},-p_{\perp}\right)$ has at $\lambda=0$ the elements

$\rho=\frac{1}{8 \pi(1+B)}\left(\frac{2 B^{\prime} W^{\prime}}{V W}-\frac{B^{\prime} V^{\prime}}{2 V^{2}}+\frac{B^{\prime \prime}}{V}+\frac{m^{2} B}{2 \xi}+\frac{B^{\prime 2}}{8 \xi B V}\right)$,

$p_{r}=\frac{1}{8 \pi(1+B)}\left(-\frac{2 B^{\prime} W^{\prime}}{V W}-\frac{B^{\prime} U^{\prime}}{2 U V}-\frac{m^{2} B}{2 \xi}+\frac{B^{\prime 2}}{8 \xi B V}\right)$,

$p_{\perp}=\frac{1}{8 \pi(1+B)}\left(\frac{B^{\prime} W^{\prime}}{V W}-\frac{2 r B^{\prime}}{W^{2}}-\frac{B^{\prime \prime}}{V}-\frac{m^{2} B}{2 \xi}-\frac{B^{\prime 2}}{8 \xi B V}\right)$.

The Gross-Pitaevskii equation (6) reads in terms of $B$

$$
\begin{aligned}
& -[(6 \xi+1) B+1]\left(\frac{B^{\prime \prime}}{2 V}+\frac{r B^{\prime}}{W^{2}}\right)+\frac{B^{\prime 2}}{4 B V}+m^{2} B(1-B) \\
& +\bar{\lambda} m^{2} B^{2}=\left(E_{0}^{2}-\frac{1}{2} \xi \mu^{2}\right) \frac{B}{U}
\end{aligned}
$$

We can now first verify that the total energy momentum tensor is conserved due to the harmonic condition (27). The singular term $B / U$ also drops out from (47) for

$$
E_{0}^{2}=8 \pi \xi \rho_{\mathrm{bi}}=\frac{1}{2} \xi \mu^{2}
$$

This was already used in (46) to cancel the $1 / U$ terms. With $\xi \equiv 2 m^{2} M_{1}^{2} / 3$ and $\bar{\mu} \equiv \mu M_{1}$ it implies again $E_{0}=$ $\bar{\mu} m / \sqrt{3}$. For very small $B \ll 1 / \xi \sim 10^{-54}$ there will be an exponential fall off, $B \sim \exp (-\sqrt{5} m r)$, so the horizon is a fraction of the Compton length thick. In this narrow range $\rho, p_{r}$ and $p_{\perp}$ vanish smoothly. In the regime $B \gg 1 / \xi$, Eq. (47) simplifies and actually reduces to Eq. (7),

$$
-\frac{B^{\prime \prime}}{V}-\frac{2 r B^{\prime}}{W^{2}}=\frac{(1-\bar{\lambda}) B-1}{2 M_{1}^{2}} .
$$

Here we can consider $B$ as vanishing sharply, $B \sim r_{h}-r$, so that $T_{B}^{\mu \nu} \neq 0$, keeping the ultimate exponential tail and decay of $\rho$ and the $p_{i}$ in mind. Eq. (46) then brings

$$
\begin{aligned}
\rho & =\frac{-1}{8 \pi(1+B)}\left(\frac{B^{\prime} U^{\prime}}{2 U V}-\frac{4+2 B+\bar{\lambda}\left(4 B+3 B^{2}\right)}{8 M_{1}^{2}}\right), \\
p_{r} & =\frac{-1}{8 \pi(1+B)}\left(\frac{2 B^{\prime} W^{\prime}}{V W}+\frac{B^{\prime} U^{\prime}}{2 U V}+\frac{6 B+3 \bar{\lambda} B^{2}}{8 M_{1}^{2}}\right),(50) \\
p_{\perp} & =\frac{1}{8 \pi(1+B)}\left(\frac{B^{\prime} W^{\prime}}{V W}-\frac{4+2 B+\bar{\lambda}\left(4 B+3 B^{2}\right)}{8 M_{1}^{2}}\right) .
\end{aligned}
$$

Let us first return to the interior where $U=2 \bar{\mu}^{2} x^{2}, V=$ $8 M_{1}^{2} x^{\prime 2} / y^{2}$ and $W=2 M_{1} x$. Eq. (49) can be written as

$$
\frac{1}{4} x^{2} B_{y y}-y B_{y}+(1-\bar{\lambda}) B=1 .
$$

To understand the structure of the problem, we again take $\lambda=0$. Then for any $A$ there is the solution

$$
B(x)=1+A y=1+A \sqrt{1-x^{2}},
$$

Expressing the shapes (50) in $y$, we have

$$
\begin{gathered}
\rho=-p_{\perp}=\frac{1}{64 \pi M_{1}^{2}(1+B)}\left(2 B+y B_{y}+4\right), \\
p_{r}=\quad \frac{-3}{64 \pi M_{1}^{2}(1+B)}\left(2 B-y B_{y}\right) .
\end{gathered}
$$

Surprisingly, their $A$-dependence factors out, keeping a vacuum equation of state $\rho=-p=3 / 64 \pi M_{1}^{2}$, so (52) is a non-uniform, exact solution of the same metric. The horizon $B=0$ is now located at $r_{h}>r_{1}$ where $y_{h}=-1 / A$. [Eq. (29) continues to negative $y \approx \sqrt{5}\left(r_{1}-r\right) / 4 r_{1}$ for $r>r_{1}$ ]. However, a problem shows up with the matching, since $W^{\prime}\left(r_{h}\right) \sim-1 / A$ cannot be of order $\bar{\mu} \sim 10^{-14}$ anymore. We thus have to deviate from the exact solution, which leads in general to a numerical problem. Analytically, this question can be considered for large $A$, by adding $1 / A^{2}$ corrections to previous solution. Expanding in $1 / A$ at fixed $s \equiv A \sqrt{5}\left(r / r_{1}-1\right) / 4$, we arrive at

$$
\begin{aligned}
B & =1-s+\frac{\sqrt{5}}{2 A} s^{2}-\frac{7 s^{3}}{6 A^{2}}+\frac{b_{1}(s)}{A^{2}} \\
\frac{U}{2 \bar{\mu}^{2}} & =1-\frac{s^{2}}{A^{2}}+\frac{u_{1}(s)}{A^{2}} \\
\frac{2}{5} V & =1-\frac{2 \sqrt{5}}{A} s+\frac{13 s^{2}}{A^{2}}+\frac{v_{1}(s)}{A^{2}} \\
\frac{W}{2 M_{1}} & =1-\frac{s^{2}}{2 A^{2}}+\frac{w_{1}(s)}{A^{2}} .
\end{aligned}
$$

The additional terms, found to be

$$
\begin{array}{ll}
b_{1}=b_{10}+b_{11} s, & u_{1}=u_{10}+4 w_{11} \ln (2-s), \\
v_{1}=v_{10}, & w_{1}=w_{10}-w_{11} \ln (2-s),
\end{array}
$$

produce an anisotropy, $\rho \neq-p_{r} \neq-p_{\perp} \neq \rho$. The horizon $B=0$ is located at $s_{h}=1+\sqrt{5} / 2 A+\mathcal{O}\left(1 / A^{2}\right)$, where $W^{\prime}=\sqrt{5}\left(w_{11}-1\right) / 2 A$. Clearly, $W^{\prime} \sim \bar{\mu}$ from (37) can be attained by tuning $w_{11}=1+3 A \bar{\mu}+O(1 / A)$. The maximum of $W$ at $s=0$ in the absence of the $w_{1}$ term has now been shifted to the horizon, which shows that the problem has a proper solution, with $A$ remaining a free parameter. $U$ has a maximum at $s=1-\sqrt{3}$. The mass seen at infinity, $M \equiv \mathcal{M}\left(r_{h}\right)=\frac{1}{2} W\left(r_{h}\right)$, coincides with $M_{1}$ to order $1 / A^{2}$. At the horizon we can fix $r_{1}$ from (36),

$$
r_{1}=M_{1}\left[1-\frac{4}{\sqrt{5} A}+\frac{4 \bar{\mu}}{\sqrt{5}}\left(2+\ln 2 \bar{\mu}^{2}\right)+\mathcal{O}\left(\frac{1}{A^{2}}\right)\right] .
$$

Properties of the solution. The groundstate occupation number becomes upon neglecting the $1 / A^{2}$ corrections

$$
N_{0}=\int \mathrm{d} \mathcal{V}\left|\Psi_{0}\right|^{2}=2 \sqrt{3} \frac{M}{m} \int_{-1 / A}^{1} \mathrm{~d} y(1+A y) .
$$

We may write the two leading orders as

$$
M=\nu N_{0} m, \quad \nu=\frac{1}{\sqrt{3}(2+A)} \approx \frac{1}{\sqrt{3} A} .
$$


Clearly, the energy $M c^{2}$ of the $\mathrm{BH}$ can be any fraction of the rest energy $N_{0} m c^{2}$ of the constituent hydrogen atoms If $\nu$ starts at a value $\nu_{c}<1$, our $\mathrm{BH}$ is likely approached in an explosive manner, possibly related to jets of quasars.

We found the sharpness of the horizon to be a fraction of the Compton length of $\mathrm{H}$. On a much larger scale $\ell_{\text {grav }}$ there is near-horizon growth of the metric functions $U / \eta \approx$ $\eta V \approx e^{\xi}$, taking place in the millimeter range,

$$
\ell_{\text {grav }}=\frac{\mathrm{d} r}{\mathrm{~d} \xi}=2 \eta M=\frac{4}{\sqrt{5}} \bar{\mu} M=4.8810^{-3} \frac{M^{2}}{M_{\star}^{2}} \mathrm{~m} .
$$

It is a realistic value, small compared to the size of the $\mathrm{BH}$, and still large compared to the Bohr radius.

The interacting situation. If the nonlinearity $\lambda$ is relevant, a numerical solution is called for. Assuming the same leading order behaviors near $r=0$, the above structure survives. Mass and particle number remain independent parameters. This analysis remains as a task for future.

Conclusion. We have questioned the general wisdom that static BHs have all their mass in the center and cannot be described by present theories. Numerical estimates show that a picture of closely packed $\mathrm{H}$ atoms naturally applies to the supermassive BH's in the center of galaxies, $M \sim M_{\star}=2.3410^{8} M_{\odot}$. We present within the Relativistic Theory of Gravitation (RTG; a colloquial term is: Not-so-General Relativity), an exact solution for a BH, of which the interior is governed by quantum matter in its Bose-Einstein condensed phase. Its density decays algebraically in the bulk and exponentially near the horizon. This solution is matched with the Schwarzschild metric, which near the horizon is deformed in RTG. Powerlaw singularities occur at the origin, that get absorbed in the Riemann description of the metric. Elsewhere, the solution is regular. The redshift at the horizon is finite, though of the order $1 / \bar{\mu} \sim 10^{14} M_{\star} / M$. To specify a $\mathrm{BH}$ requires not only the mass (and, in general, charge and spin), but also the rest energy of the constituent matter.

Our BH is a quantum fluid confined by its own gravitation. In the interior, time keeps its standard role. No Planckian physics is involved; Hawking radiation is absent and Bekenstein-Hawking entropy plays no role.

Our BH has one "hair". As one would expect for a classical theory of gravitation, when the quantum matter in the $\mathrm{BH}$ has reached a certain groundstate, the classical metric allows the system still to go to a lower energy state. Indeed, the passage of celestial bodies will induce oscillations in the metric and emission of gravitational waves, which, upon re-equilibration, increase the binding energy, finally up to $100 \%$ of the rest energy of its constituents, $N m c^{2}$. This property may explain the enormous jets and energy output of quasars and also be responsible for very high energy cosmic rays, $E>410^{19} \mathrm{eV}$. [15]

An important question is whether formation of realistic supermassive BHs brings the matter indeed in or near the Bose-Einstein condensed groundstate. Also the stability of the solution needs to be studied. It also remains to be seen whether the phenomenological value for the parameter $\xi$ has a microscopic underpinning. Extension to finite temperatures, not presented here, will exhibit a $T^{3 / 2}$ fraction of thermal atoms.

Calculation of the normal mode spectrum may lead to predictions that deviate from the ones of GTR; this spectrum may be observed in the foreseeable future.

If we apply Eq. (48) to Logunov's case $\rho_{\mathrm{bi}}<0$, it follows that $\xi<0$, so $B<0$ due to (18). To avoid a singularity in e.g. (46), a lower bound $B(0)>-1$ will be required. As already indicated by the exact solution (52), $B$ will then go to zero at some point well below $M$. This prevents fitting to the external metric and excludes our BH solution.

We failed to apply our approach to GTR, technically because it lacks compensation for the singular $1 / U$ terms. If no other solution exists for the considered physical situation, GTR must be abandoned and replaced by another theory, RTG being the first candidate. In view of its smaller symmetry group, this may have far reaching consequences for singularities in classical gravitation and for quantum approaches to gravitation, while Minkowski space-time needs no quantization.

$* * *$

The author has benefited from discussion with Steve Carlip, Kostas Skenderis and Bahar Mehmani.

\section{REFERENCES}

[1] G. Chapline, E. Hohlfield, R. B. Laughlin and D. I. Santiago, Philos. Mag. B 81, 235 (2001).

[2] I. Dymnikova, Int. J. Mod. Phys. D 12, 1015 (2003).

[3] P. O. Mazur and E. Mottola, Proc. Nat. Acad. Sci. 101, 9545 (2004).

[4] R. J. McLure and J. S. Dunlop, Mon. Not. Roy. Astron. Soc. 331, 795 (2002).

[5] A. D. Sacharov, Sov. Phys. JETP 22, 241 (1966).

[6] A.A. Logunov, The Theory of Gravity, (Nauka, Moscow, 2001).

[7] S.S. Gershtein, A.A. Logunov and M.A.Mestvirishvili, Phys. Uspekhi 49, 1179 (2006).

[8] Th. M. Nieuwenhuizen, Europhys. Lett. 78, 10010 (2007); In Quantum Theory, Recons. of Found. - 4, ed. G. Adenier et al., AIP Conf. Proc. 962 (Melville, 1997), pp 149.

[9] L. D. Landau and E. M. Lifshitz, The Classical Theory of Fields, (Pergamon, Oxford, U.K., 1951; revised 1979).

[10] S. V. Babak and L. P. Grishchuk, Phys. Rev. D61, 024038 (1999)

[11] L. P. Pitaevskii and S. Stringari, Bose-Einstein Condensation, (Oxford University Press, Oxford, 2003).

[12] N. D. Birrell and P. C. W. Davies, Quantum Fields in Curved Space, (Cambridge Univ. Press, New York, 1994).

[13] H.T.C. Stoof, J. M. V. A. Koelman and B. J. Verhaar, Phys. Rev. B 38, 4688 (1988).

[14] R. Ruffini and S. Bonazzola, Phys. Rev. 187 (1969) 1767; M. Colpi et al., Phys. Rev. Lett. 57 (1986) 2485.

[15] Press release of the Auger collaboration: http://www. auger.org/news/PRagn/AGN_correlation_more.html 